\title{
MRI for Pretreatment Lymph Node Staging in Uterine Cervical Cancer
}

\author{
Hyuck Jae Choi ${ }^{1}$ \\ Seung Hyup Kim² \\ San-Soo Seo ${ }^{1}$ \\ Sokbom Kang ${ }^{1}$ \\ Sun Lee ${ }^{1}$ \\ Joo-Young Kim ${ }^{1}$ \\ Young Hoon Kim ${ }^{1}$ \\ Jong Seok Lee ${ }^{1}$ \\ Hyun Hoon Chung ${ }^{1}$ \\ Joo-Hyuk Lee ${ }^{1}$ \\ Sang-Yoon Park ${ }^{1}$
}

Keywords: cancer, lymph nodes, MRI, oncologic imaging, paraaortic lymph node, pelvic lymph node, uterine cervical cancer

\section{DOI:10.2214/AJR.05.0263}

Received February 15, 2005; accepted after revision June 7, 2005.

1Department of Radiology, Research Institute and Hospital, National Cancer Center, 809 Madu 1-dong, Ilsan-gu, Koyang, Kyonggi, Korea 411-769. Address correspondence to S. Y. Park.

2Department of Radiology and Institute of Radiation Medicine, Seoul National University College of Medicine, Seoul, Korea.

WEB

This is a Web exclusive article.

\section{AJR 2006; 187:W538-W543}

0361-803X/06/1875-W538

(C) American Roentgen Ray Society

OBJECTIVE. The purpose of this article is to assess the accuracy of MRI in detecting pelvic and paraaortic lymph node metastasis from uterine cervical cancer using various imaging criteria.

CONCLUSION. Although MRI analysis resulted in relatively low sensitivity, size and margin (spiculated or lobulated) were useful criteria for predicting lymph node metastasis from cervical cancer.

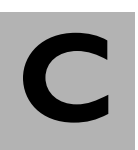

ervical cancer is the second most frequently diagnosed malignancy in women worldwide, and it is the only major gynecologic malignancy clinically staged according to International Federation of Obstetrics and Gynecology (FIGO) recommendations [1]. Clinical staging of cervical cancers is accurate in only approximately $60 \%$ of cases, which is far less than surgical staging accuracy $[2,3]$. Lymph node metastasis is not a factor for FIGO staging; however, nodal metastases in gynecologic malignancies have an adverse impact on survival, especially in cases of paraaortic node involvement in cervical cancer $[4,5]$. Although nodal resection before radiation therapy results in a higher survival rate in patients with grossly enlarged pelvic and paraaortic lymph nodes $[6,7]$, routine pretreatment surgical staging is not recommended. For this reason, inaccurate pretreatment assessment of lymph node involvement can lead to suboptimal treatment [8, 9].

CT and MRI have been used to assess paraaortic and pelvic lymph nodes in patients with cervical cancer. A meta-analysis of such studies concluded that these methods have only moderate sensitivity and specificity for detecting metastases [10]. These studies relied on the size and shape of lymph nodes, and the analyses were based on region-specific comparisons. It was reported that margin and appearance are valid criteria for assessing lymph node metastasis from rectal cancer [11]. However, to our knowledge, no reports define the validity of criteria other than size and shape, and no reports have described node-by-node comparisons in the detection of metastatic pelvic lymph nodes in patients with uterine cervical carcinoma.

The purpose of this study was to assess the accuracy of MRI in detecting metastatic lymph nodes in cervical cancer patients using various imaging criteria.

\section{Materials and Methods \\ Patients and Staging Workup}

Patients included in this retrospective study were those with histopathologically confirmed FIGO stages IB-IVA invasive cervical cancer, which was determined by a conventional workup that included MRI. Patients were recruited between October 2001 and October 2004, ranged in age from 18 to 65 years (mean age, 48 years), had no contraindications to the surgical procedure, had no evidence of distant metastases, and had an Eastern Cooperative Oncology Group performance status of $0-1$. Patients with small cell carcinoma $(n=2)$ and patients who were not to undergo laparoscopic lymph node dissection $(n=63)$ were excluded.

After histologic confirmation of invasive cervical carcinoma, the FIGO stage was determined using bimanual pelvic examination, excretory urography, sigmoidoscopy, cystoscopy, colposcopy, and radiographic examination of the lungs.

Fifty-five patients were classified into two groups according to FIGO staging. The first group consisted of patients with stages IB1 (tumor size $\leq 4 \mathrm{~cm}$ ) and IIA disease, and they underwent a conventional lymphadenectomy combined with a radical hysterectomy via laparotomy $(n=23)$. The second group consisted of patients classified with stages IB2 (tumor size $>4 \mathrm{~cm}$ ) and IIB and greater disease, and they received chemoradiation preceded by a laparoscopic paraaortic and pelvic lymphadenectomy $(n=32)$. 


\section{MRI for Pretreatment of Uterine Cervical Cancer}
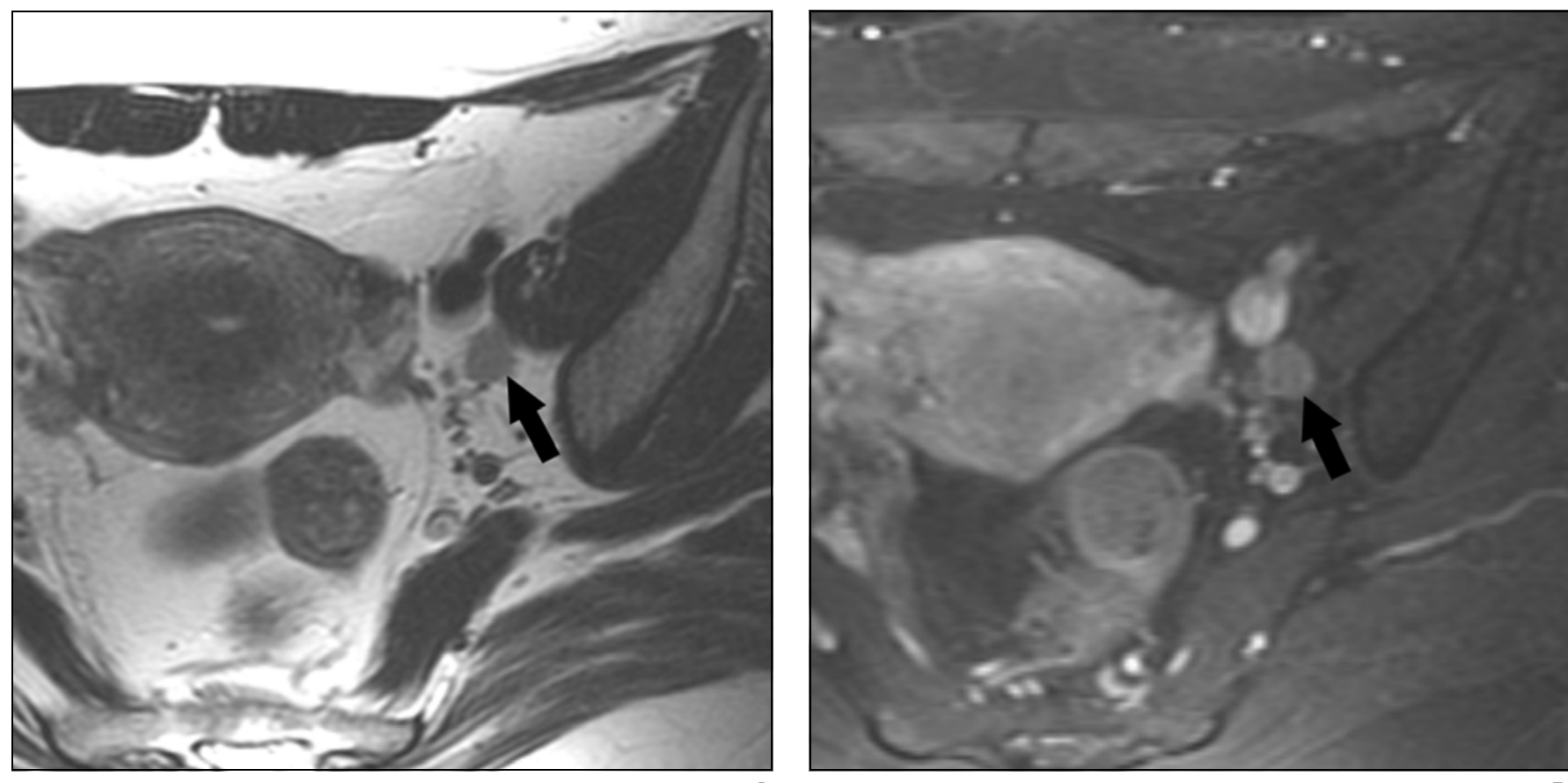

A

Fig. 1-37-year-old woman with true-positive left internal iliac lymph node metastasis in stage IIB uterine cervical cancer.

A, MR axial T2-weighted fast spin-echo image (TR/TE, 5,000/68; echo-train length, 21) shows an ovoid lymph node (arrow, 11-mm short-axis diameter) with spiculated border in left internal iliac area.

B, Gadolinium-enhanced axial T1-weighted turbo spin-echo sequence image (175/4.2; echo-train length, 3) shows heterogeneous enhancement of left internal node (arrow). Histopathology showed one positive node in left internal iliac and obturator area of six lymph nodes sampled.
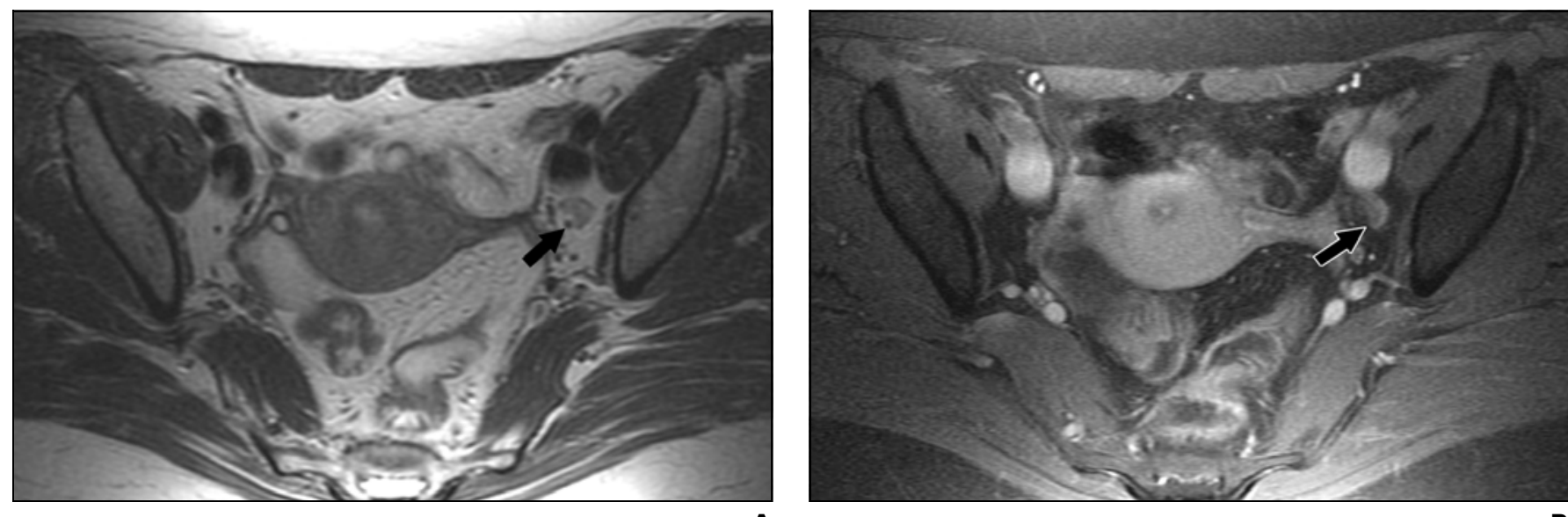

A

Fig. 2-50-year-old woman with true-positive pelvic lymph node metastases in stage IIIA uterine cervical cancer.

A, MR axial T2-weighted fast spin-echo image (TR/TE, 5,000/68; echo-train length, 21) shows elongated lymph node (arrow, 5-mm short-axis diameter) with lobulated margin in left internal iliac area.

B, Gadolinium-enhanced axial T1-weighted turbo spin-echo sequence image (175/4.2; echo-train length, 3) shows heterogeneous enhancement of left internal iliac lymph node (arrow). Histopathology showed one positive node in left internal iliac and obturator area of four nodes sampled.

Informed consent was obtained from all patients before the laparoscopic paraaortic and pelvic lymphadenectomy was performed via laparotomy or laparoscopy. The institutional review board approved this trial.

\section{MRI Protocol}

MRI was performed on the Signa 1.5-T system (GE Healthcare) using a pelvic array coil for the pelvic scan and a torso phased-array coil for the paraaortic scan. Scans were obtained using the fol- lowing parameters for the pelvic region: axial T1weighted fast spin-echo sequence (TR/TE, 600/10; slice thickness, $5 \mathrm{~mm}$; interslice gap, $2 \mathrm{~mm}$; field of view, $24 \times 24 \mathrm{~cm}$; matrix, $256 \times 192$; echo-train length, 4 ; signals acquired, 3 ; no fat saturation; band- 


\section{Choi et al.}
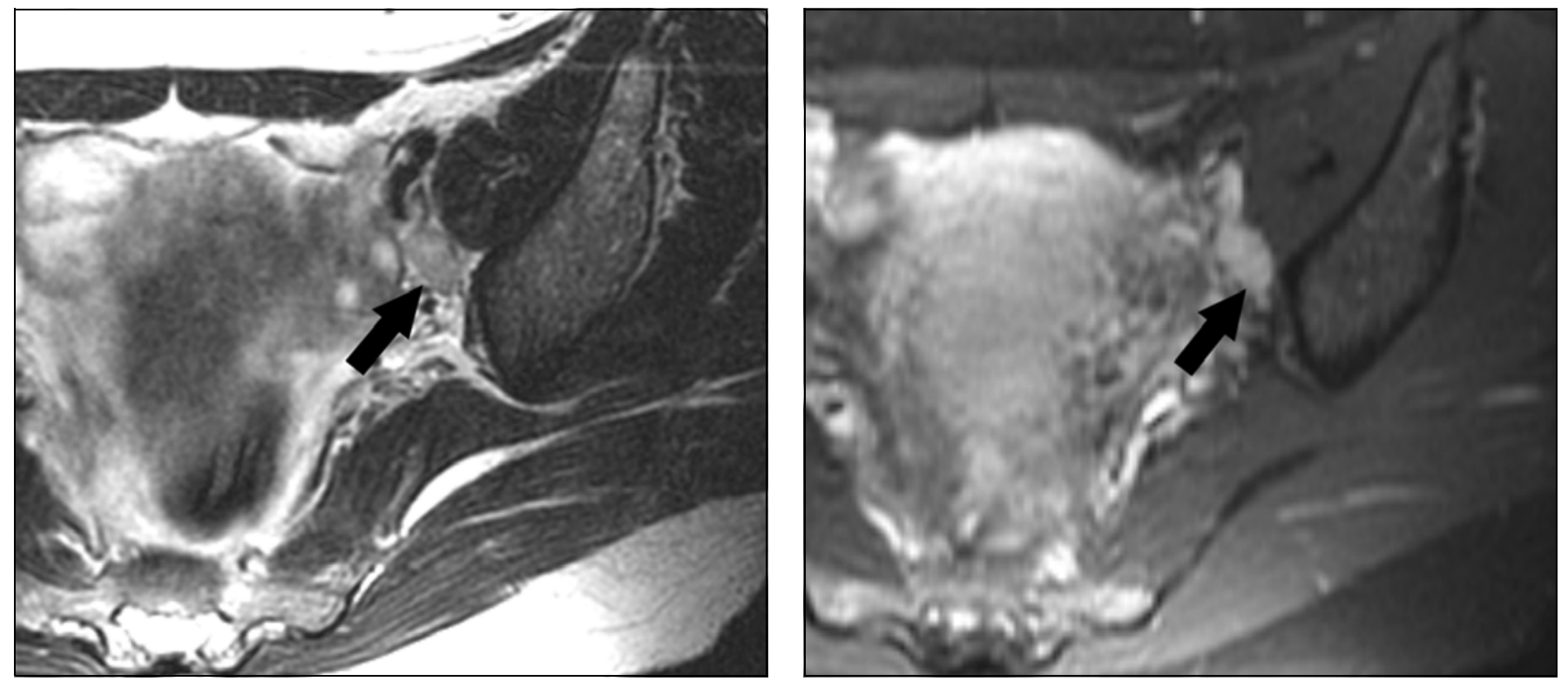

A

Fig. 3-48-year-old woman with true-positive left internal iliac lymph node metastasis in stage IIB uterine cervical cancer.

A, MR axial T2-weighted fast spin-echo image (TR/TE, 5,000/68; echo-train length, 21) shows ovoid lymph node (arrow, 10-mm short-axis diameter) with smooth margin in left internal iliac area.

B, Gadolinium-enhanced axial T1-weighted turbo spin-echo sequence image (175/4.2; echo-train length, 3) shows homogeneous enhancement of left internal iliac lymph node (arrow). Histopathology showed one positive node in left internal iliac and obturator area of four nodes sampled.

width, $31.25 \mathrm{kHz}$ ), axial T2-weighted fast spin-echo sequence (5,000/68; slice thickness, $3 \mathrm{~mm}$; interslice gap, $1 \mathrm{~mm}$; field of view, $24 \times 24 \mathrm{~cm}$; matrix, $25 \times$ 192; echo-train length, 21 ; signals acquired, 4 ; no fat saturation; bandwidth, $31.25 \mathrm{kHz}$ ), sagittal T2weighted fast spin-echo sequence $(5,000 / 68$; slice thickness, $3 \mathrm{~mm}$; interslice gap, $3 \mathrm{~mm}$; field of view, $24 \times 24 \mathrm{~cm}$; matrix, $256 \times 192$; echo-train length, 26; signals acquired, 4; no fat saturation; bandwidth, $31.25 \mathrm{kHz}$ ), coronal T2-weighted fast spin-echo sequence $(5,000 / 68$; slice thickness, $3 \mathrm{~mm}$; interslice gap, $3 \mathrm{~mm}$; field of view, $24 \times 24 \mathrm{~cm}$; matrix, $256 \times 192$; echo-train length, 26; signals acquired, 4; no fat saturation; bandwidth, $31.25 \mathrm{kHz}$ ), gadolinium-enhanced axial and sagittal T1-weighted turbo spin-echo sequence (175/4.2; slice thickness, $5 \mathrm{~mm}$; interslice gap, $2 \mathrm{~mm}$; field of view for axial plane, $24 \times 24 \mathrm{~cm}$; field of view for sagittal plane, $24 \times 24$ cm; matrix, $256 \times 192$; echo-train length, 3; signals acquired, 2; fat saturation; bandwidth, $31.25 \mathrm{kHz}$ ). For the paraaortic region, an axial fast spin-echo T2weighted sequence with 16 seconds of breath-holding (2,000/68; slice thickness, $8 \mathrm{~mm}$; interslice gap, $2 \mathrm{~mm}$; field of view, $32 \times 24 \mathrm{~cm}$; matrix, $256 \times 160$; echo-train length, 20; signals acquired, 1 ; no fat saturation; bandwidth, $31 \mathrm{kHz}$ ) was performed. Ten patients did not undergo gadolinium injection because they were recruited between March 2004 and October 2004, during which time we did not perform gadolinium-enhanced T1-weighted MRI.

\section{Surgical Technique}

All patients underwent preoperative bowel preparations for 2 days, were given prophylactic antibiotics, and were operated on using identical instruments and techniques. Two expert laparoscopic surgeons performed all operations and were aware of the MRI findings before lymph node dissection. After a thorough exploration of the pelvic and abdominal cavities, paraaortic lymph node dissection and pelvic lymph node dissection were performed via laparotomy or laparoscopic surgery. The upper limit of the paraaortic lymph node dissection was the duodenum, and the margin of node dissection was demarcated using endoclips; these margins were confirmed using abdominal radiographs after surgery.

\section{Classification of Lymph Node Regions}

Lymph nodes were grouped into seven regions according to anatomic landmarks: right paraaortic (including right and left paraaortic lymph nodes), both common iliac, both external iliac, and both internal iliac and obturator areas.

\section{Histopathologic evaluation}

Histopathologic evaluation of the lymph nodes was the diagnostic standard. Thin sections were cut, stained with $\mathrm{H}$ and $\mathrm{E}$, and examined microscopically by a pathologist. Each lymph node was sliced at 2-mm intervals perpendicular to the longest diameter to maximize the likelihood of detect- ing micrometastases. The total number of lymph nodes harvested in each region was recorded.

\section{Analysis}

MR images were assessed by two radiologists with 3 and 5 years of experience in gynecologic imaging, including MRI. They interpreted MR images of pelvic lymph nodes and were unaware of the histologic results. The first step involved a thorough inspection of all visible lymph nodes. The characteristics of each lymph node detected on MR images were recorded in terms of short-axis diameter, ratio of long-to-short-axis diameter (round [ratio $<1.2$ ], oval [ratio 1.2-1.5], elongated [ratio $>1.5$ ]), margin (smooth, lobulated, spiculated), and enhancement pattern (homogeneous, heterogeneous). Axial T2weighted fast spin-echo sequences were used to investigate the short-axis diameter, ratio of long-toshort-axis diameter, and margin. Gadolinium-enhanced axial and sagittal T1-weighted turbo spinecho sequences were used to investigate the enhancement pattern. Lymph node diameter measurements were made using electronic calipers with a PACS monitor.

When a positive lymph node was detected in the pelvic region during MRI and the pelvic region was also positive for a metastatic node during the surgical-pathologic examination, the node detected on MRI was regarded as a true-positive lymph node (Figs. 1-3). In addition, when a pelvic region had 
Fig. 4-False-positive pelvic lymph node metastases in 44-year-old woman with stage IIB uterine cervical cancer.

A and B, MR T2-weighted fast spin-echo axial (A) and coronal (B) images (TR/TE, 5,000/68; echo-train length, 21) show elongated lymph node (arrow, 8-mm short-axis diameter) with spiculated margin in right internal iliac area. Histopathology showed no positive nodes in right internal iliac and obturator area of four nodes sampled.
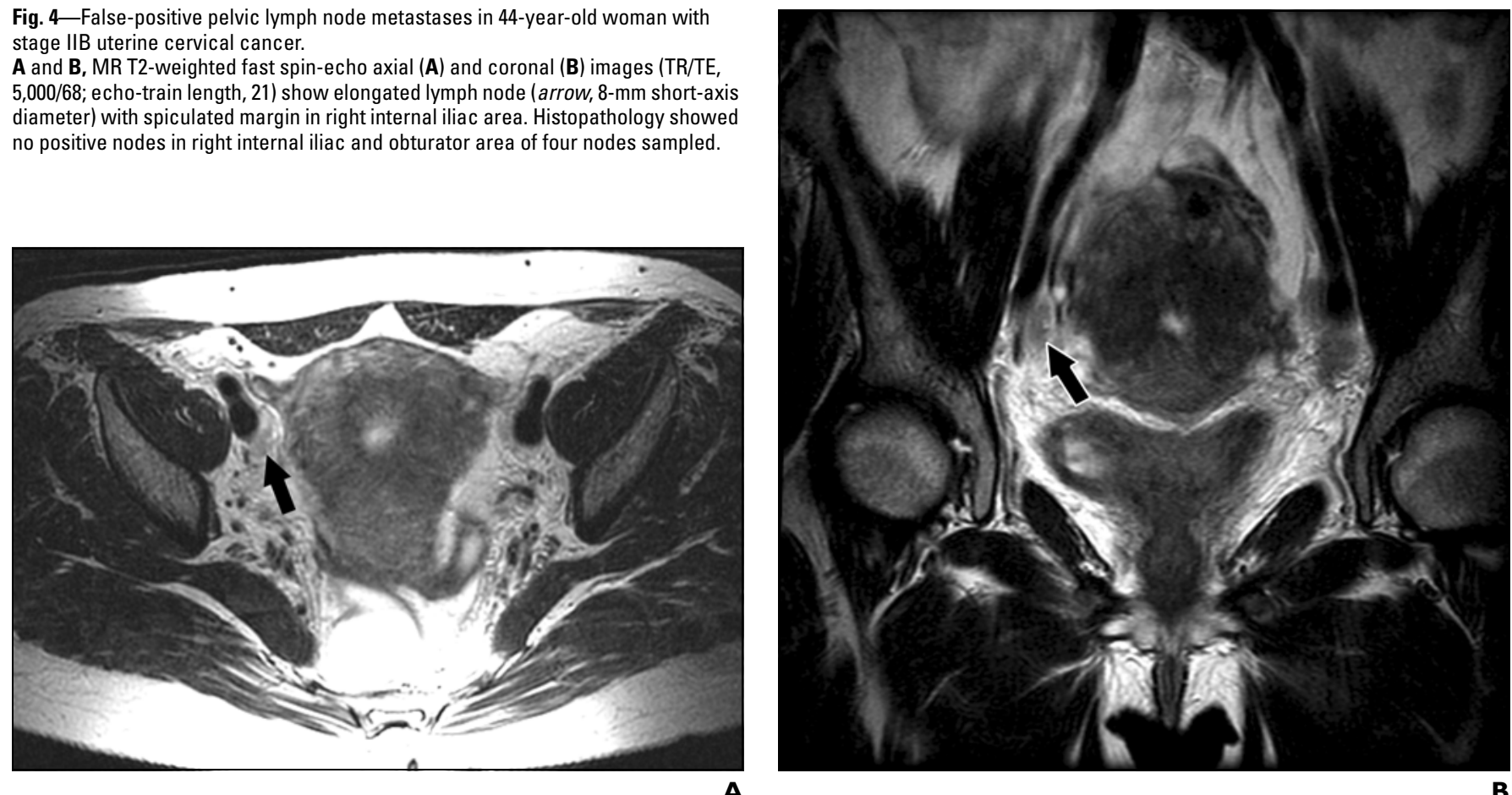

A

more than one positive node on MRI and when the region was positive for metastatic nodes according to surgical-pathologic examination, the positive nodes seen on MR images were all regarded as true-positive nodes unless the number of nodes seen on MR images exceeded the number of positive nodes determined by surgical-pathologic examinations. When the number of positive lymph nodes seen on MR images exceeded the number of positive nodes determined by histopathologic examination, we regarded the larger nodes seen on MRI as positive. Nodes seen on MR images were regarded as false-positives when a pelvic region was determined to have a positive lymph node on MRI, but the region was negative according to the surgical-pathologic examination (Fig. 4). Conversely, nodes seen on MR images were regarded as false-negatives when a pelvic region was found to be negative for metastatic lymph nodes on MR images, but was found to be positive according to surgical-pathologic examination.

\section{Statistics}

The sensitivity, specificity, positive predictive value, and accuracy of each criterion in the prediction of nodal positivity were calculated. Independent Student's $t$ tests were used to compare the size of metastatic and nonmetastatic lymph nodes. The Pearson's chi-square test was used to compare the margins and enhancement patterns. A $p$ value of less than 0.05 was considered to indicate a significant difference.

\section{Results \\ Histopathology}

Histopathologic examination revealed metastatic lymph nodes in 17 (30.9\%) of the 55 patients. These nodes appeared in $36(9.4 \%)$ of 385 regions, and $62(3.2 \%)$ metastatic nodes were found out of 1,909 nodes examined. During surgery, the number of lymph nodes sampled in each patient ranged from 9 to 68 (mean, 34.7). Histopathologic examination showed that metastatic lymph nodes were found in one $(5.3 \%)$ of 19 patients with stage IB1 disease; two $(66.7 \%)$ of three patients with stage IB2 disease; two (33.3\%) of six patients with stage IIA disease; 10 (43.5\%) of 23 patients with stage IIB disease; and in two $(50 \%)$ of four patients with stage IIIA, IIIB, or IVA disease.

\section{Number, Size, and Shape of Lymph Nodes on MRI}

MRI showed 86 lymph nodes in total. Of the lymph nodes visible on MRI, the mean short-axis diameter of metastatic lymph nodes $(9.3 \pm 3.0 \mathrm{~mm}, n=27)$ was larger than that of nonmetastatic lymph nodes $(7.5 \pm 4.2 \mathrm{~mm}$, $n=59)(p=0.027)$. The prediction of nodal status was most accurate when a size criterion greater than $9 \mathrm{~mm}$ was applied to the shortaxis diameter of the lymph node. Table 1 provides the sensitivity, specificity, positive pre- dictive value, and accuracy for both node-bynode and region-specific comparisons. Only one $(14.3 \%)$ of seven metastatic paraaortic lymph nodes was detected on MRI with a short-axis diameter of $11.9 \mathrm{~mm}$. The ratio of long-to-short-axis diameter was similar for both positive and negative lymph node groups $(p=0.263)$ (Table 2).

\section{Margin of Lymph Node}

The presence of a lymph node with a lobulated $(n=20)$ or a spiculated $(n=8)$ border among 86 detectable lymph nodes on MRI showed sensitivity and positive predictive values of $21.0 \%$ and $46.4 \%$, respectively, and they were reliable indicators of lymph node metastasis $(p=0.044)$ (Table 2). Among them, six lymph nodes that had a lobulated $(n=4)$ or spiculated $(n=2)$ border were metastatic lymph nodes measuring less than $9 \mathrm{~mm}$ in short-axis diameter.

\section{Enhancement Pattern}

Gadolinium-enhanced T1-weighted MRI scans was performed on 45 of 55 patients, and 69 metastatic pelvic lymph nodes were found in this group. Although heterogeneous enhancement was more commonly seen in metastatic lymph nodes (17/24 [70.8\%]) than in nonmetastatic lymph nodes (21/45 [46.7\%]), this difference was not found to be statisti- 


\section{Choi et al.}

TABLE I: Accuracy Profiles of Size Criterion

\begin{tabular}{l|c|c|c|c|c|c|c|c}
\hline $\begin{array}{c}\text { Short-Axis } \\
\text { Diameter of } \\
\text { Lymph Nodes } \\
(\mathrm{mm})\end{array}$ & \multicolumn{3}{|c|}{ Node-by-Node Comparison } & \multicolumn{4}{c}{ Region-Specific Comparison } \\
\cline { 2 - 8 } & Sensitivity & Specificity & PPV & Accuracy & Sensitivity & Specificity & PPV & Accuracy \\
\hline All visible & 43.5 & 96.8 & 31.4 & 95.1 & 58.3 & 88.3 & 33.9 & 85.5 \\
lymph nodes & & & & & & & & \\
4 & 41.9 & 97.1 & 32.5 & 95.3 & 52.8 & 88.5 & 32.2 & 85.2 \\
5 & 40.3 & 97.7 & 36.8 & 95.8 & 50.0 & 88.8 & 31.6 & 85.2 \\
6 & 37.1 & 98.1 & 39.7 & 96.1 & 44.4 & 92.6 & 38.1 & 88.1 \\
7 & 33.9 & 98.5 & 43.8 & 96.4 & 44.4 & 93.4 & 41.0 & 88.8 \\
8 & 29.0 & 99.0 & 50.0 & 96.8 & 38.9 & 95.7 & 48.3 & 90.4 \\
9 & 24.2 & 99.1 & 57.7 & 97.0 & 33.3 & 97.4 & 57.1 & 91.4 \\
10 & 16.1 & 99.6 & 58.8 & 96.9 & 19.4 & 98.0 & 50.0 & 90.6 \\
\hline
\end{tabular}

Note—All data are percentages. PPV = positive predictive value.

TABLE 2: Lymph Node Characteristics on MRI

\begin{tabular}{l|c|c|c|c}
\hline \multirow{2}{*}{\multicolumn{1}{c|}{ MRI Findings }} & \multicolumn{4}{|c}{ No. (\%) of Lymph Nodes } \\
\cline { 2 - 5 } & Total & Metastatic & Nonmetastatic & $p$ \\
\hline Shape & $18(20.9)$ & $6(7.0)$ & $12(14.0)$ & \\
$\quad$ Round & $25(29.1)$ & $11(12.8)$ & $14(16.3)$ & \\
Oval & $43(50)$ & $10(11.6)$ & $33(38.4)$ & \\
$\quad$ Elongated & & & & 0.044 \\
Margin & $58(67.4)$ & $14(16.3)$ & $44(51.2)$ & \\
$\quad$ Smooth & $20(23.3)$ & $9(10.5)$ & $11(12.8)$ & \\
Lobulated & $8(9.3)$ & $4(4.7)$ & $4(4.7)$ & \\
$\quad$ Spiculated & & & & \\
Enhancement pattern & & $7(10.1)$ & $24(34.8)$ & \\
$\quad$ Homogeneous & $31(44.9)$ & $17(24.6)$ & $21(30.4)$ & \\
Heterogeneous & $38(55.1)$ & &
\end{tabular}

${ }^{a}$ Gadolinium-enhanced T1-weighted MR scans were obtained only for pelvic areas in 45 of 55 patients in this study, and there were 69 metastatic pelvic lymph nodes in those patients.

cally significant $(p=0.076)$ (Table 2$)$. The sensitivity and positive predictive value of this criterion were $24.6 \%$ and $44.7 \%$, respectively. Six lymph nodes with heterogeneous enhancement patterns were metastatic, measuring less than $9 \mathrm{~mm}$ in short-axis diameter.

\section{Discussion}

The presence of a metastatic lymph node radically modifies the prognosis and treatment of cervical cancer patients $[4,5,12,13]$. However, the presence of lymph node metastasis does not change the clinical FIGO stage of cervical cancer [14].

The accuracy and sensitivity rates for MRI are reported to be between $76 \%$ and $100 \%$ and $36 \%$ and $89.5 \%$, respectively [15-22]. In the present study, which used the criterion for metastasis as a lymph node with a short-axis diameter of $9 \mathrm{~mm}$ or larger, the accuracy and sensitivity rates for region-specific comparisons were $91.4 \%$ and $33.3 \%$, respectively. This sensitivity rate of $33.3 \%$ is lower than those reported by others. A reason for this may be that the present study examined transverse slices made at 2-mm intervals, whereas lymph nodes are conventionally examined in only one or two longitudinal sections. Thus, we may have detected metastases that might have been missed using conventional techniques. In addition, the range and completeness of surgical staging were different from other studies in that previous studies did not perform systematic surgical staging, including paraaortic dissection for all cases [15-22].

The only generally accepted MRI criterion in the diagnosis of a metastatic pelvic lymph node is node size [15-22]. Diameter limits ranging from 6 to $15 \mathrm{~mm}$ have been applied, with $10 \mathrm{~mm}$ being the most common criterion for the upper limit of a normal lymph node [15-22]. In the current study, a short-axis diameter of $9 \mathrm{~mm}$ was the optimal value using MRI, not only on a region-by-region basis but also on a node-by-node basis.

The present study found that lobulated or spiculated lymph node margins, according to MR images, were strong predictors of lymph node metastasis. These types of margins could be caused by a desmoplastic reaction or tumor infiltration into the perinodal fat. Interestingly, this margin criterion helped in the identification of metastatic lymph nodes with a short-axis diameter of less than $9 \mathrm{~mm}$, with six of 12 such nodes detected using this parameter. The sensitivity and positive predictive value on MRI to detect lymph node metastasis was $33.9 \%$ and $56.8 \%$, respectively, when both of these criteria were appliedthat is, a short-axis diameter of $9 \mathrm{~mm}$ for the upper limit of a normal lymph node and a spiculated or lobulated margin.

The usefulness of the long-to-short-axis ratio as a criterion is unclear in most other reports, and Yang et al. [22] reported an accuracy rate of $77.3 \%$ when using the long-toshort-axis diameter ratio on MR images. In the present study, application of the long-toshort-axis ratio criterion resulted in low accuracy. This may be because the axial MR images are not likely to show the maximum cross section of lymph nodes.

MRI of the neck has shown that heterogeneous signal intensity on contrast-enhanced T1-weighted images is a good indicator of metastatic nodes [23, 24]. Heterogeneous enhancement of lymph nodes could be the result of tumor infiltration of the lymph nodes, necrosis, or a mucin pool, but in the present study, application of the enhancement pattern criterion did not distinguish metastatic from nonmetastatic nodes. However, use of this criterion resulted in the detection of six additional (of 12) metastatic nodes with short-axis diameters of less than $9 \mathrm{~mm}$. We believe that a difference in imaging might be associated with a difference in histopathology. We suggest that despite the statistical insignificance of the findings according to enhancement pattern in this study (which may have been because of the small number of patients who had undergone contrast-enhanced T1-weighted MRI), these findings may be helpful for a differential diagnosis.

In the present study, the criteria of central necrosis and fat hilum were not applied be- 


\section{MRI for Pretreatment of Uterine Cervical Cancer}

cause gadolinium-enhanced images were acquired with fat saturation, and it was possible that central necrosis and fat hilum could not be distinguished.

A limitation of the current study was that some assumptions were made in the node-bynode comparisons. With these assumptions, it was possible that smaller or nondetectable lymph nodes were metastatic and larger or detectable lymph nodes were nonmetastatic. Obtaining the histology of individual nodes identified on cross-sectional images is likely to remain challenging. Another limitation was that although size and margin were useful criteria on MRI, metastatic and nonmetastatic lymph nodes significantly overlapped in these areas. A third notable limitation was that surgeons were aware of the MRI findings before lymph node dissections, and this may have added verification bias. Finally, the MRI parameters for the paraaortic region were different from those of the pelvic region, with both slice thickness and interslice gap being larger compared with those of T2-weighted images for the pelvic region. This may partly account for the lower sensitivity in detecting metastatic paraaortic lymph nodes compared with metastatic pelvic lymph nodes.

In conclusion, we found that an increased lymph node short-axis diameter correlated with lymph node metastasis in uterine cervical cancer patients. Furthermore, the presence of a spiculated or lobulated lymph node border was also predictive of metastasis. However, the sensitivity of MRI was relatively low, not only in node-by-node comparisons but also in region-specific comparisons.

\section{References}

1. Parkin DM, Bray F, Ferlay J, Pisani P. Estimating the world cancer burden: Globocan 2000. Int J Cancer 2001; 94:153-156

2. Ballon SC, Berman ML, Lagasse LD, Petrilli ES, Castaldo TW. Survival after extraperitoneal pelvic and paraaortic lymphadenectomy and radia- tion therapy in cervical carcinoma. Obstet Gynecol 1981; 57:90-95

3. Avrette HE, Dudan RC, Ford JH. Exploratory celiotomy for surgical staging of cervical cancer. Am J Obstet Gynecol 1972; 113:1090-1096

4. Takeshima N, Yanoh K, Tabata T, Nagai K, Hirai Y, Hasumi K. Assessment of the revised International Federation of Gynecology and Obstetrics staging for early invasive squamous cervical cancer. $G y$ necol Oncol 1999; 74:165-169

5. Tanaka Y, Sawada S, Murata T. Relationship between lymph node metastases and prognosis in patients irradiated postoperatively for carcinoma of the uterine cervix. Acta Radiol Oncol 1984; 23:455-459

6. Downey GO, Potish RA, Adcock LL, Prem KA, Twiggs LB. Pretreatment surgical staging in cervical carcinoma: therapeutic efficacy of pelvic lymph node resection. Am J Obstet Gynecol 1989; 160:1055-1061

7. Potish RA, Twigg LB, Okagaki T, Prem KA, Addcock LL. Therapeutic implications of the natural history of advanced cervical cancer as defined by pretreatment surgical staging. Cancer $1985 ; 56: 956-960$

8. Lagasse LD, Creasman WT, Shingleton HM, Ford JH, Blessing JA. Results and complications of operative staging in cervical cancer: experience of the Gynecologic and Oncology Group. Gynecol Oncol 1980; 9:90-98

9. Kupets R, Covens A. Is the International Federation of Gynecology and Obstetrics staging system for cervical carcinoma able to predict survival in patients with cervical carcinoma? An assessment of clinimetric properties. Cancer 2001; 92:796-804

10. Scheidler J, Hricak H, Yu KK, Subak L, Segal MR. Radiological evaluation of lymph node metastases in patients with cervical cancer: a meta-analysis. JAMA 1997; 278:1096-1101

11. Kim JH, Beets GL, Kim MJ, Kessels AG, Beets-Tan RG. High-resolution MR imaging for nodal staging in rectal cancer: are there any criteria in addition to the size? Eur J Radiol 2004; 52:78-83

12. Chung CK, Nahhas WA, Zaino R, Stryker JA, Mortel R. Histologic grade and lymph node metastasis in squamous cell carcinoma of the cervix. Gynecol Oncol 1981; 12:348-354
13. Van Nagell JR Jr, Roddick JW Jr, Lowin DM. The staging of cervical cancer: inevitable discrepancies between clinical staging and pathological findings. Am J Obstet Gynecol 1971; 110:973-978

14. Kamura T, Tsukamoto N, Tsuruchi N, et al. Multivariate analysis of the histopathologic prognostic factors of cervical cancer in patients undergoing radical hysterectomy. Cancer 1992; 69:181-186

15. Outwater E, Kressel HY. Evaluation of gynecologic malignancy by magnetic resonance imaging. $R a$ diol Clin North Am 1992; 30:789-806

16. Kim SH, Kim SC, Choi BI, Han MC. Uterine cervical carcinoma: evaluation of pelvic lymph node metastasis with MR imaging. Radiology 1994; 190:807-811

17. Togashi K, Nishimura K, Itoh K, et al. Uterine cervical cancer: assessment with high-field MR imaging. Radiology 1986; 160:431-435

18. Togashi K, Nishimura K, Sagoh T, et al. Carcinoma of cervix: staging with MR imaging. Radiology 1989; 171:245-251

19. Waggenspack GA, Amparo EG, Hanninan EV. MR imaging of uterine cervical carcinoma. $J$ Comput Assist Tomogr 1988; 12:409-414

20. Choi SH, Kim SH, Choi HJ, Park BK, Lee HJ. Preoperative magnetic resonance imaging staging of uterine cervical carcinoma: results of prospective study. J Comput Assist Tomogr 2004; 28:620-627

21. Yu KK, Hricak H, Subak LL, Zaloudek CJ, Powell CB. Preoperative staging of cervical carcinoma: phased array coil fast spin-echo versus body coil spin-echo T2-weighted MR imaging. AJR 1998; 171:707-711

22. Yang WT, Lam WW, Yu MY, Cheung TH, Metreweli C. Comparison of dynamic helical CT and dynamic MR imaging in the evaluation of pelvic lymph nodes in cervical carcinoma. AJR 2000; 175:759-766

23. Som PM. Detection of metastasis in cervical lymph nodes: CT and MR criteria and differential diagnosis. AJR 1992; 158:961-969

24. van den Brekel MW, Castelijins JA, Stel HV, et al. Detection and characterization of metastatic cervical adenopathy by MR imaging: comparison of different MR techniques. J Comput Assist Tomogr 1990; 14:581-589 